\title{
O estudo das operações formais no processo de projeto do arquiteto Peter Eisenman
}

\author{
The study of formal tools in Peter Eisenman's design process
}

\author{
Andressa Carmo Pena Martinez \\ Universidade Federal de Viçosa, Brasil \\ andressamartinez@gmail.com \\ Denise Mônaco dos Santos \\ Universidade Federal de Viçosa, Brasil \\ demonaco@sc.usp.br
}

\author{
Douglas Lopes de Souza \\ Universidade Federal de Viçosa, Brasil \\ dglopes@gmail.com \\ Caio Magalhães Castriotto \\ Universidade Federal de Viçosa, Brasil \\ caio.castriotto@gmail.com
}

\begin{abstract}
This paper presents part of the research on the study of formal operations in Peter Eisenman's design process. The initial hypothesis is the possibility of translating the architect's design process and his formal tools, into a shape grammar, in selected works. In this sense, the research aims to simulate these formal tools through digital models, analyze the variations in the Diagram of Interiority and Exteriority phases; understand the design process using Rhinoceros software and its interaction with grasshopper, in search of the decoding and interpretation of a system of rules implicit in the architect's design process.
\end{abstract}

Keywords: Diagrams; Design Process; Formal tools; Shape Grammars; Peter Eisenman.

\section{Introdução}

Peter Eisenman consolidou uma produção arquitetônica de formas complexas, através de um processo projetual caracterizado pelo método dos diagramas e suas ferramentas formais. A produção do arquiteto é comumente dividida em fases, sendo as três primeiras definidas como (1) Diagramas de Anterioridade (Fase de investigação teórica), (2) Diagramas de Interioridade (Grids, Forms e Bars), (3) Diagramas de Exterioridade, quando também ocorre a transição para os meios digitais.

A primeira fase, denominada Diagramas da Anterioridade, caracteriza-se por uma produção essencialmente teórica que se iniciou durante a pesquisa para a sua tese de doutorado, defendida em 1963. Grande parte dos estudos iniciais de Eisenman dedicaram-se aos tratados propostos por Vitruvius, Alberti, Leonardo da Vinci, bem como análises dos processos de concepção da forma nas villas palladianas, nas obras de Le Corbusier, Giuseppe Terragni, dentre outros. Esses estudos, com influências "da terminologia do linguista estruturalista Noam Chomsky" (Somol in Eisenman, 2001, p.15, nossa tradução), tinham em comum a busca pela compreensão de uma linguagem arquitetônica baseada na análise de diferentes sistemas de geração da forma.

Neste processo investigativo na fase dos Diagramas de Anterioridade, chamou-nos atenção a presença das mesmas bases teóricas, das quais derivaram a gramática da forma. Segundo Correia, Duarte e Leitão (2012), as gramáticas generativas de Noam Chomsky (1957) foram uma das bases para o desenvolvimento da gramática da forma por Stiny e Gips, em 1972. "A partir deste trabalho original, de Stiny e Gips, a aplicação das gramáticas formais para interpretação e avaliação de trabalhos de arte gerou uma gama mais ampla da teoria da gramática da forma e suas aplicações" (Correia et al., 2012, p.490, nossa tradução). Derivam também destes estudos a gramática paramétrica da forma (Stiny, 1980), as gramáticas descritivas (Stiny, 1981), dentre outras.

Também nos pareceu pertinente pontuar a possível influência dos tratados de Vitruvius, dos postulados da arquitetura clássica de Alberti e Palladio e, mesmo posteriormente, dos princípios arquitetônicos defendidos por Le Corbusier, como um percurso investigativo de Eisenman na busca por um sistema de concepção arquitetônica, que teria rebatimento posterior na formulação do seu processo do diagrama e as ferramentas formais. Segundo Kwon (2003), Stiny e Mitchel (1978) também estudaram profundamente uma série de regras para as villas projetadas pelo arquiteto Andrea Palladio, que culminaram na extração de uma gramática formal. Essa gramática das villas palladianas focou na descrição de 72 regras para a geração das plantas-baixas, com um grid inicial retangular, utilizado para a criação das paredes externas e a compartimentação interna.

De modo análogo, a pesquisa de Coutinho et al. (2011) comprovou a decodificação de uma gramática da forma extraída do tratado de Alberti "De re aedificatoria" que, inicialmente considerado uma descrição, comprovou-se ser "um texto sem ilustrações porque descreve um sistema de regras, em vez de um modelo de soluções" (Coutinho et al., 2011, p.789, nossa tradução). José Duarte, um dos condutores desta pesquisa, utilizou uma metodologia "combinando a gramática descritiva de Stiny (1981) com as gramáticas da forma para produzir descrições semânticas de processos de projetos". (Beirão, Duarte, 2005, pg.493, nossa tradução).

Neste sentido, a investigação apresentada neste artigo parte da hipótese inicial de que o processo de projeto do arquiteto Peter Eisenman através dos diagramas tinha como um dos 
pressupostos a construção de um processo generativo, baseado em um sistema de regras a partir da criação de ferramentas formais, à semelhança das bases teóricas investigadas pelo arquiteto na fase dos Diagramas de Anterioridade (fase de investigação teórica).

Pretendemos, então, simular esse processo de projeto em exemplares selecionados de Peter Eisenman através do desenvolvimento de modelos digitais no software Rhinoceros e sua interação com o Grasshopper, com o objetivo de traduzir em algoritmos este sistema de regras utilizados pelo arquiteto, em busca da sistematização de uma possível gramática da forma.

\section{Gramática da Forma}

As "gramáticas da forma são sistemas generativos, baseados em regras, que permitem capturar, criar e compreender processos de projeto", (Correia et al., 2012, p. 491, nossa tradução). Segundo Correia et al. (2012), cada regra da gramática da forma é codificada como uma operação de transição que modifica a forma do objeto de um estado para o outro. Em geral, todas as estratégias de interpretação das gramáticas são baseadas na expansão recursiva dos estados, "de uma forma inicial a uma final, e a ordem em que cada estado é expandido, determina a estratégia da investigação. No entanto, quando o estágio final é atingido, torna-se necessário conhecer o percurso seguido. Ao final, cada estado é enriquecido com informação adicional, nomeando que estado e que operador de transição foi utilizado para gerá-lo." (Correia et al., 2012,p. 491, nossa tradução).

De modo semelhante, o processo de projeto de Eisenman, a partir dos diagramas, constitui um sistema generativo de regras ou operações formais, que "trariam uma série de movimentos, como um jogo de xadrez, no qual cada movimento é uma resposta ao último estado" (Eisenman, 2001, pg.74, nossa tradução). Segundo Eisenman (2001), por exemplo, um objeto A pode se transformar em um diferente objeto $B$, através da criação de estados intermediários. Estes estados intermediários são conectados sequencialmente em um processo não linear, não descritivo e, em alguns casos, não explicitado pelo arquiteto.

Nesta pesquisa consideramos como operadores de transição as ferramentas formais de Eisenman, aplicadas sobre as regras a serem descobertas e explicitadas em alguns projetos utilizados como estudos de caso, através do desenvolvimento de modelos digitais no software Rhinoceros e sua interação com o Grasshopper.

\section{Metodologia}

Com o objetivo de simular o processo de projeto de Peter Eisenman através do desenvolvimento de modelos digitais, os procedimentos adotados foram: (1) reprodução das ferramentas formais definidas pelo arquiteto, aqui compreendidas como os operadores de transição de forma, e sua tradução em algoritmos no Grasshopper; (2) seleção de três projetos do Peter Eisenman utilizados como estudos de caso; (3) desenvolvimento de modelos digitais dos exemplares selecionados no Rhinoceros e Grasshopper; (4) análise e interpretação dos algoritmos gerados no Grasshopper, com o objetivo de explicitar e sistematizar as operações formais, ou seja, o sistema de regras intrínsecos ao processo projetual do arquiteto; (5) comparação entre os sistemas de regras emergentes em cada projeto, com o objetivo de encontrar repetições, similaridades e diferenças entre os projetos escolhidos; (6) teste e exploração do sistema computacional de geração formal, a partir da manipulação de outros parâmetros, com o objetivo de simular novos resultados de projeto dentro da mesma gramática da forma aferida.

\section{Reprodução das ferramentas formais definidas pelo arquiteto}

Utilizando elementos primários para composição (ponto, linha e planos), uma malha (grid) como suporte virtual para a gramática compositiva e o cubo como forma inicial, foram organizados os scripts de 23 operações formais definidas pelo arquiteto, como extrusão, torção, extensão, entrelaçamento, deslocamento, desmontagem, dentre outras. Este primeiro conjunto já contribuiu para a formação de uma grande gama de composições formais e geometrias complexas, que foram reproduzidas no Grasshopper.

Algumas operações como entrelaçamento, interferência, empenamento, transferência, deslizamento, dentre outras, combinam duas ou mais ferramentas de transformação da forma e só foram compreendidas a partir da etapa seguinte, de modelagem dos projetos selecionados.

\section{Seleção de três projetos de Peter Eisenman}

Dentre o vasto repertório de projetos do arquiteto, inicialmente adotou-se como recorte temporal a fase analógica dos Diagramas de Interioridade, caracterizada principalmente pela produção de casas (série Houses I a $X I$ ), uma vez que constituem objetos arquitetônicos em menor escala, utilizados por Eisenman como experimentação inicial de seu processo projetual diagramático. A fase dos Diagramas de Interioridade (1967-1977) caracterizou-se pela produção arquitetônica em que as ferramentas formais consideram apenas o objeto arquitetônico em si; o comportamento do conjunto de regras não possui influência de condicionantes externas à edificação.

Dentre estas, selecionamos a House VI (1972-1975) e House $X$ (1975). A primeira destaca-se como um dos primeiros produtos resultantes do processo generativo do sistema de operações formais do arquiteto. Na verdade, antes da House $I V$, os diagramas eram elaborados após a concepção do objeto arquitetônico, como meio de justificativa da forma gerada. No entanto, é na House VI que o processo de Eisenman se consolida e o projeto é resultante do diagrama em si. Segundo Eisenman (2001), a partir da casa VI, os diagramas moveram-se de ícones da interioridade euclidiana para índices de uma geometria topológica, desenhada como euclidiana, devido às limitações dos métodos de desenho analógicos existentes na época.

A House $X$ foi selecionada por possuir duas formas iniciais o cubo e o ponto da el-form (forma L), marcando a transição entre os projetos da fase inicial do plano cartesiano e da 
interioridade euclidiana, para as relações topológicas estabelecidas nas el-forms, ambas pertencentes ainda à fase do Diagrama de Interioridade.

O último projeto utilizado como estudo de caso, a Casa Guardiola (1988), foi selecionado por utilizar os diagramas da House $X$ como um texto inicial. Ambas se enquadram na produção denominada como el-forms ou formas em L, ou seja, possuem semelhanças na forma inicial adotada, o que sugere similaridades nos processos de geração formal, pressupondo-se a análise comparativa dos sistemas de regras entre os três exemplares, ao final da pesquisa.

A Casa Guardiola também pode ser considerada como o primeiro marco da transição para a fase dos Diagramas de Exterioridade. Esta fase, além de marcar a transição para a produção digital do arquiteto, acrescenta parâmetros sitespecific, ou seja, as condicionantes externas que estabelecem relações à priori com a forma (estrutura, lugares, textos, dentre outras), influenciando diretamente a produção arquitetônica de Eisenman. Seu processo incorpora uma primeira ideia de um modelo digital em três dimensões, mas, acima disso, ele adiciona a primeira ideia do local específico em que a obra será alocada: o primeiro vislumbre dos diagramas de exterioridade.

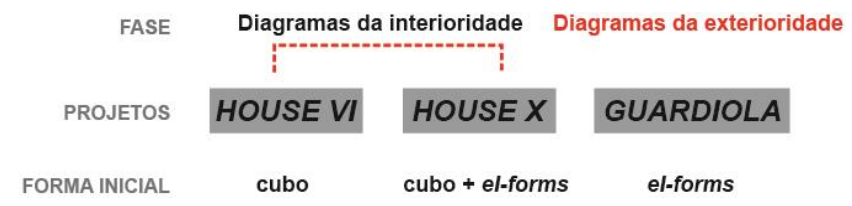

Figura 1: Esquema ilustrativo dos três projetos selecionados para investigação.

\section{Resultados}

Apresentamos aqui, na sequência em que foram desenvolvidos, os modelos digitais produzidos no software Rhinoceros e sua interação com o Grasshopper de apenas duas obras selecionadas: A Casa Guardiola e a House VI. Como a pesquisa ainda está na fase de decodificação e interpretação do sistema de regras, apresentamos parte do processo de explicitação do processo projetual.

Na fase de concepção da gramática da forma, utilizaremos como método três estágios semelhantes aos apresentados por Correia et al. (2012), em seu estudo sobre a interpretação computacional do tratado de Alberti "De re aedficatoria": (a) um organograma descritivo que explicita a ordem e as operações desenvolvidas no processo de cada um dos projetos; (b) uma aproximação deste organograma para extração das regras e proporções definidas em cada estágio; (c) a sistematização da gramática.

Por ora, apresentamos apenas o "estágio a" (organograma descritivo) e parte do "estágio b" (extração das regras) dos projetos selecionados.

\section{A Casa Guardiola (1988)}

Segundo Eisenman (2001), o projeto da Casa Guardiola possui como ferramenta formal o imprinting (impressão, tradução nossa) e a ferramenta conceitual, tracing (traço, tradução nossa). Do mesmo modo, o desenvolvimento do modelo digital no software Rhinoceros através da interação com os algoritmos no grasshopper indicou a existência de duas operações formais principais: (1) interseção > deslocamento, no estado intermediário de geração formal, que nomeamos como 'A', e (2) deslocamento > união, no estado que nomeamos como 'B'. O resultado final do projeto é a união de ambos os estados da forma, que possuem percursos de geração semelhantes. Entre elas são aplicadas sucessivas rotações: em ' $A$ ', essencialmente relacionadas ao ponto de referência da el-form e em 'B', à mudança de quadrante no plano cartesiano. Tem-se as seguintes regras generativas em ambos os estados intermediários da forma, conforme ilustrado no diagrama da figura 2:

\section{forma inicial: El-form ou forma em L}

estado ' $A$ ' operação formal 1: interseção > deslocamento; derivações da operação formal 1, denominadas como operação formal 2 > deslocamento > rotação; ou interseção > rotação > escala > deslocamento;

estado 'B' operação formal 3: deslocamento > união; e derivações da operação 3 , denominadas como operação formal 4: deslocamento > espelho > rotação > união.

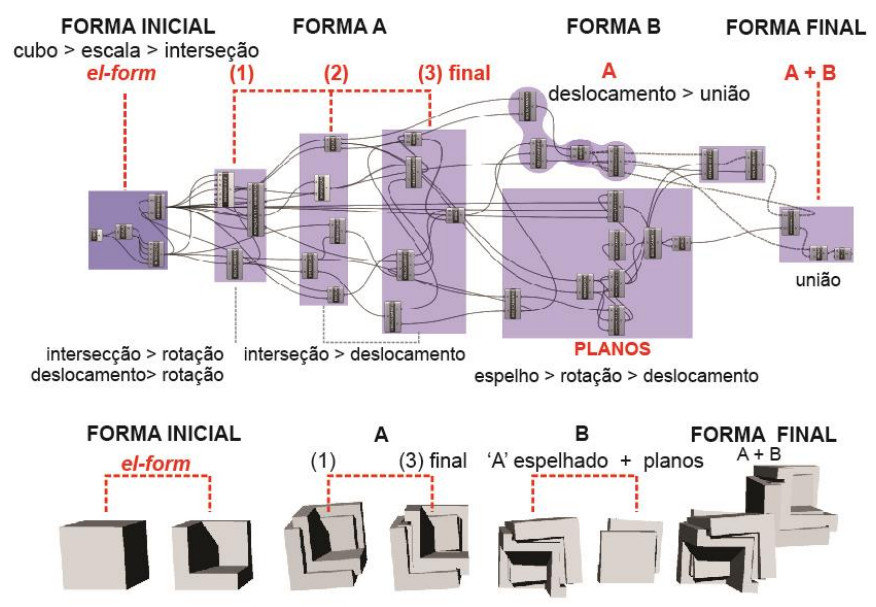

Figura 2: Esquema das operações formais no grasshopper e a modelagem, no software Rhinoceros, dos estados intermediários correspondentes da Casa Guardiola,

Sistematizamos o organograma descritivo do processo projetual em escala de cinza, criando a correspondência entre os estados intermediários da forma e as operações formais correspondentes, conforme ilustrado pela figura 3 . 


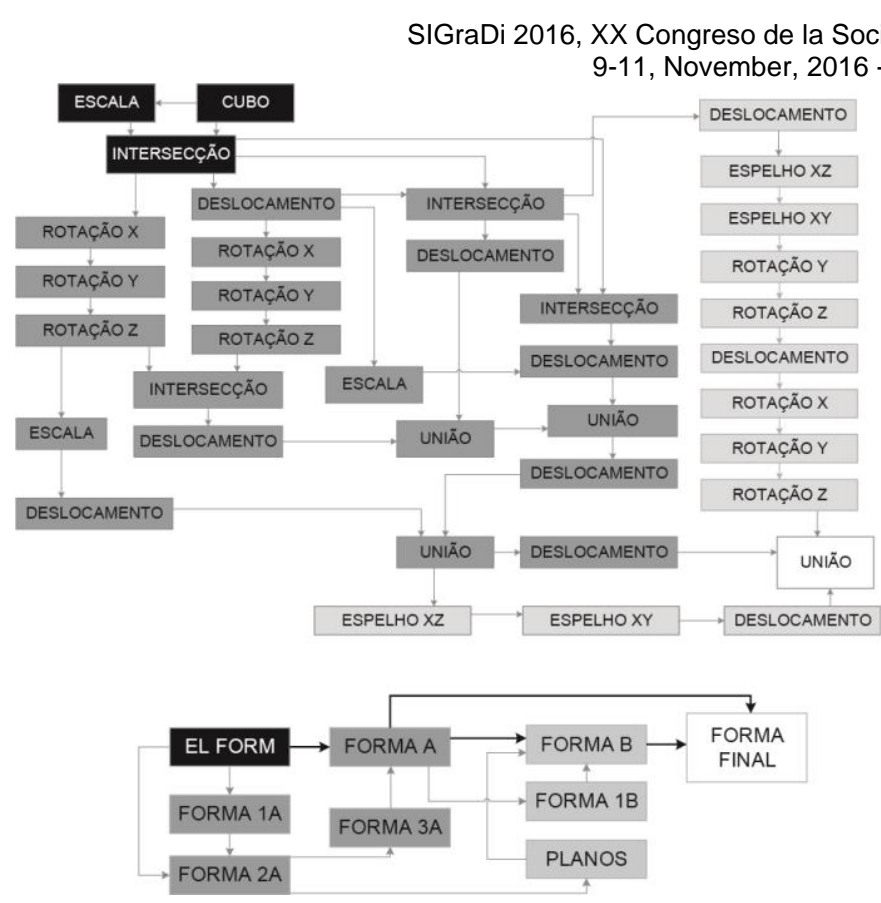

Figura 3: Organograma esquemático relacionando as operações formais, aos estados intermediários da forma na Casa Guardiola.

\section{A House VI (1972-1975)}

Segundo Eisenman, a House VI possui a ferramenta formal slippage (deslizamento, tradução nossa) e duas ferramentas conceituais denominadas inversion (inversão, tradução nossa) e montage (montagem, tradução nossa). Mas, segundo 0 arquiteto, esse projeto também inaugura um processo chamado decomposição, que culminará mais adiante na House $X$.

O desenvolvimento do modelo digital indicou a existência de seis sequencias de operações formais principais: extensão > deslocamento; divisão > interseção; divisão > deslocamento; escala > deslocamento; divisão > extensão e extensão > deslocamento, conforme ilustrado nos diagramas da figura 4 :

\section{forma inicial ou estado 1: cubo}

estado 2: decomposição e deslocamento dos planos, gerados pelo grid da forma inicial cúbica.

estado 3: operação formal 1: extensão > deslocamento;

estado 4: operação formal 2: divisão > interseção;

estado 5: operação formal 3: divisão > deslocamento; operação formal 4: escala > deslocamento;

estados 6 e 7: operação formal 5: divisão > extensão; operação formal 6: extensão > deslocamento

De modo semelhante à Casa Guardiola, sistematizamos o organograma descritivo do processo projetual em escala de cinza, criando a correspondência entre os estados intermediários da forma $e$ as operações formais correspondentes, conforme ilustrado pela figura 5. A forma final é a união dos estados 4, 5, 6 e 7.

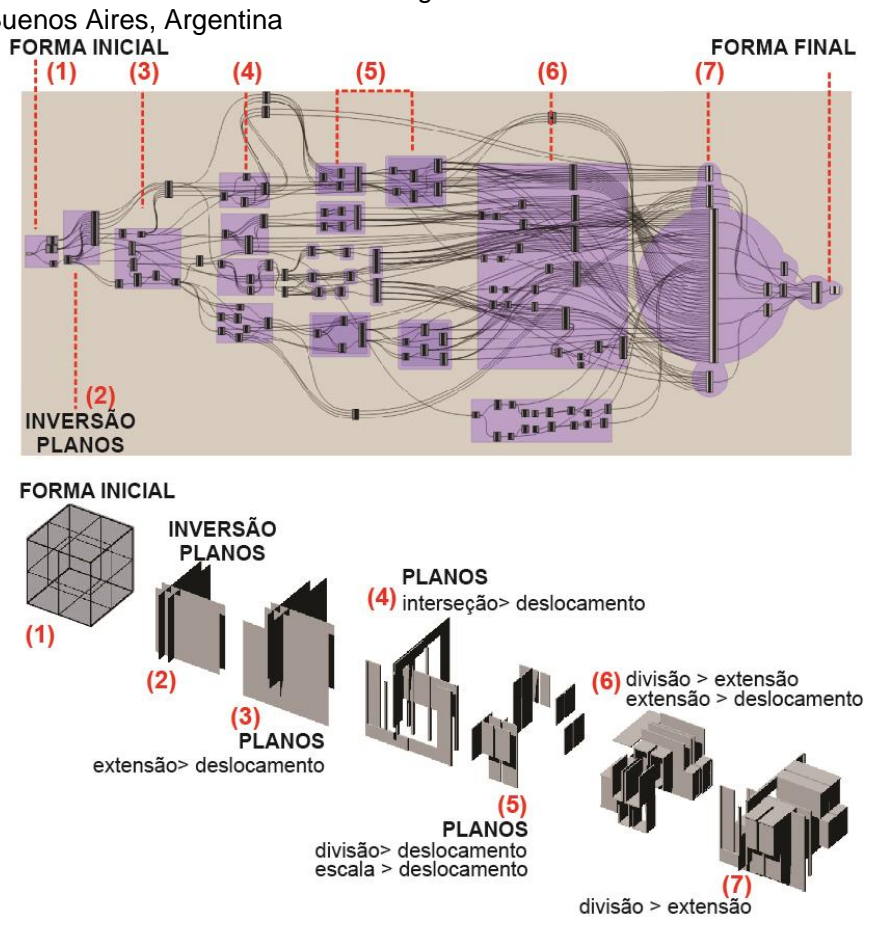

Figura 4: Esquema das operações formais como algoritmos do grasshopper e a modelagem dos estados intermediários correspondentes, na House VI.

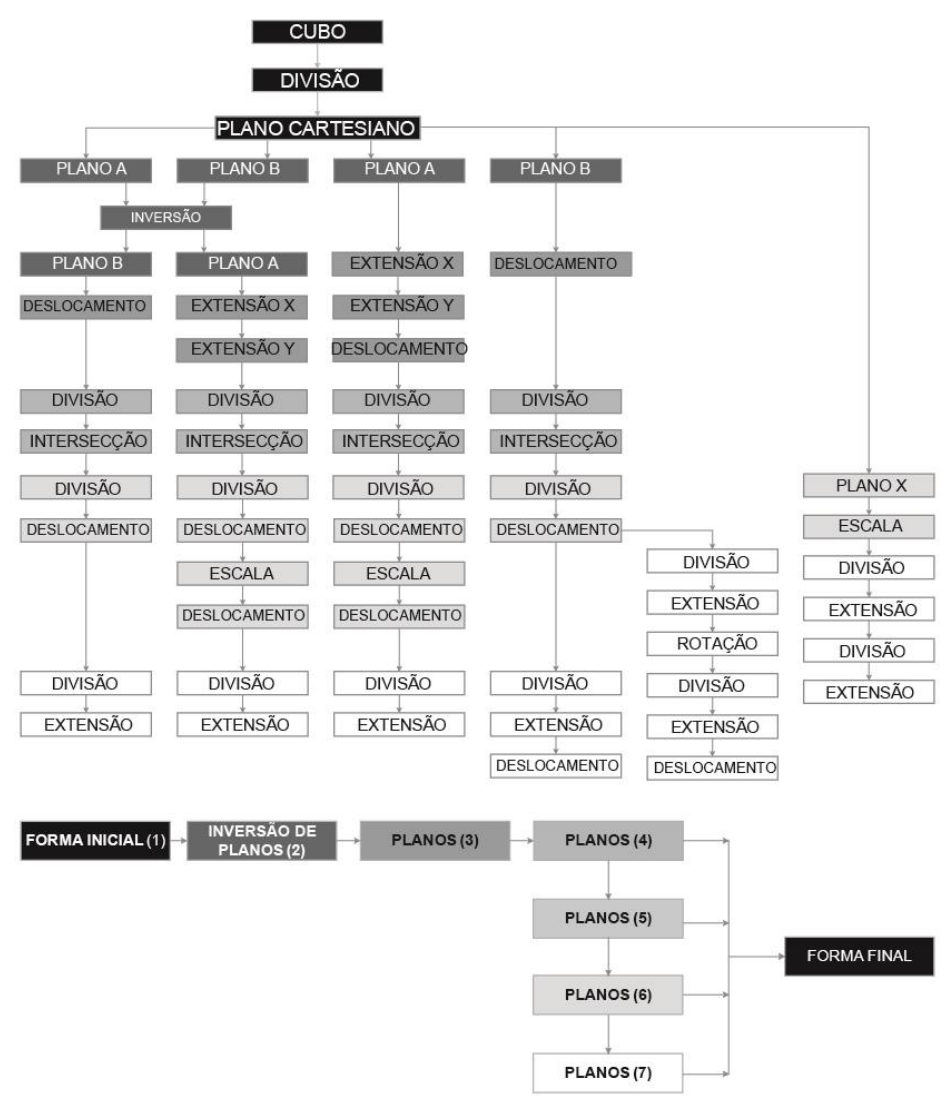

Figura 5: Organograma esquemático relacionando as operações formais aos estados intermediários da forma na House VI. 


\section{Conclusão}

A modelagem dos dois projetos como ponto de partida e a sua organização cronológica, indicam preliminarmente a simplificação no processo projetual de Peter Eisenman ao longo do tempo. Na House VI, por exemplo, as decisões formais estão menos explícitas do que no projeto da Casa Guardiola, na década posterior, o que se reflete também na simplificação do algoritmo no Grasshopper neste último projeto. A inserção do site-specific e outros inputs, na fase do Diagrama da Exterioridade também auxiliam a explicitação das decisões projetuais.

Durante o percurso da pesquisa e modelagem da House $X$, percebemos ser igualmente importante a modelagem da House $X I$, uma vez que ela se enquadra completamente no grupo das el-forms e conclui a série de casas House I a House XI.

Vislumbra-se com a atual pesquisa, além da aferição de uma gramática da forma para cada um dos projetos selecionados, também a tradução dos algoritmos em sistemas generativos para a simulação de novos projetos, com linguagens semelhantes, a partir da alteração de parâmetros.

Em pesquisas futuras, também consideramos pertinente traçar uma análise comparativa entre projetos de formas iniciais semelhantes dentro da produção da fase Diagramas de interioridade: Grids, Forms e Bars. A investigação parcial já demonstrou que a House VI, situada na fase de grids, tem a concepção volumétrica através de planos, enquanto a Casa Guardiola, característica da série el-forms (ou formas em L), já apresenta um processo projetual distinto. A comparação entre projetos com formas iniciais semelhantes e a distinção entre estes, enriquecerão também estudos futuros.

\section{Agradecimentos}

À FAPEMIG Fundação de Amparo à Pesquisa do Estado de Minas Gerais pelo apoio à divulgação científica e participação no evento. Ao CNPq, financiador da bolsa de iniciação científica vinculada a este projeto de pesquisa.

\section{Referências}

Beirão, J.; Duarte, J. (2005, Sept 21). Urban Grammars: Towards Flexible Urban Design. In: 23th eCAADe Conference, Proceedings... (pp. 491-499), Lisboa: eCAADe

Correia, R.; Duarte, J.; Leitão, A. (2012, Sept 12). Gramatica: A general $3 d$ shape grammar interpreter targeting the mass customization of housing. In: 30th eCAADe Conference, Proceedings... (pp. 489-496), Praga: eCAADe.

Costa, E.; Coutinho, F.; Duarte, J.; Kruger, M. (2012, Sept 12). Modelling Aberti's Column System: Generative modelling and digital fabrication of classical architectural elements. In: 30th eCAADe Conference, Proceedings... (pp. 459-467), Praga: eCAADe.

Coutinho, F.; Costa, E.; Duarte, J.; Kruger, M. (2011). A computacional interpretation of "De re aedificatoria". Translating Alberti's column system into a shape grammar. In: 29th eCAADe Conference, Proceedings... (pp. 789-798), Ljubljana: eCAADe.

Davidson, C. (Ed.) (2006). Tracing Eisenman: Peter Eisenman Complete Works. New York: Rizzoli.
(1999). Diagram Diaries. London: Thames\&Hudson.

(2004). Eisenman Inside Out: Selected Writings 1963-1988.

New Haven/London: Yale University Press.

(2007). Written into the Void. Selected Writings 1990-2004. New Haven/London: Yale University Press.

Karle, D.; Kelly, B. M. (2011, Mar 10). Parametric Thinking. In: ACADIA, Regional - Parametricism: Student Performance Criteria, University of Nebraska-Lincoln College of Architecture. Proceedings... (pp. 109-113), Nebraska: ACADIA.

Kwon, D. (2003). ArchiDNA: A Generative System for Shape Configuration. Master's thesis in Architecture. University of Washington.

Scheeren, R.; Sperling, D. M. (2013, Nov. 20). Dos Diagramas aos Parâmetros: Transformações no Design Digital. In: Sigradi 2013 (pp. 262-265), Valparaiso: Sigradi. 